\title{
Edge Detection using Skewed and Elongated basis Functions
}

\author{
S. Anand \\ Assistant Professor \\ Mepco Schlenk Engineering \\ College \\ Sivakasi
}

\author{
S. Jeeva \\ P.G Student \\ Mepco Schlenk Engineering \\ College \\ Sivakasi
}

\author{
T. Thivya \\ P.G Student \\ Mepco Schlenk Engineering \\ College \\ Sivakasi
}

\begin{abstract}
This paper proposed an edge detection using Skewed and Elongated Basis functions (SEBF). In an image, edges present at any directions and any scale. Common edge detection algorithms are single scale, and limited in directions. The proposed method efficiently removes these difficulties by using SEBF that are having multiscale and non separable properties. The two dimensional non separable properties of SEBF provide multiple directions, whereas the multiscale to explore the hidden edge information at various scales. The SEBF are derived from anisotropic directionlets are applied for edge detection to effectively preserving edges in all orientations and scales. This paper uses Figure of Merit (F), accuracy, sensitivity, specificity characteristics to analyze the performances. The experimental results showed that the proposed algorithms provide improvement in all performance measure.
\end{abstract}

\section{Keywords}

Edge Detection, Directionlet Basis, Scale Multiplication

\section{INTRODUCTION}

Edge detection plays an important role in computer vision and image analysis. Edges are the abrupt change points in an image that are basic features and change points give the locations of the image contour [1]. The edge representation of an image reduces the amount of data to be processed, and it retains important information about the shapes of objects in the scene. This description of an image is easy to integrate into a large number of recognition algorithms [2]. Many classical edge detectors have developed over time. Sobel, Prewitt, Canny are the common classical edge detection operators [3]. They are based on the principle of matching local image segments with specific edge patterns. The edge detection is realized by the convolution with a set of directional derivative masks. They are all defined on a 3 by $3-$ pattern grid, so they are efficient and easy to apply. Sobel, Prewitt used as local gradient operators to detect the edges having certain orientations and performed poorly when the edges were blurred and noisy. In certain situations where the edges are highly directional, some edge detector works well because, their patterns fit the edges better way and they are not suitable for complex (natural) images.

The one-dimensional (1-D) wavelet transform (WT) has become very successful in the last decade because it provides a good multiresolution representation of 1-D piecewise smooth signals [4], [5]. The application of wavelets to image processing requires the design of two dimensional (2-D) wavelet bases. The most common approach is to construct such bases using 2-D separable filter-banks, which consist of the direct product of two independent 1-D filter-banks in horizontal and vertical directions. Filtering with high-pass filters with enough vanishing moments along these two directions leads to a sparse representation of smooth signals. This method is conceptually simple and has very low complexity while all the 1-D wavelet theory carries over. Despite their success, the standard separable 2-D WT fails to provide a sparse representation in the presence of 1-D discontinuities, like edges or contours. These discontinuities, being highly anisotropic objects present in images, are characterized by a geometrical coherence that is not properly captured by the standard isotropic WT.

The anisotropic basis functions can "match" anisotropic objects Fig.1 (b). However, ensuring an efficient matching between anisotropic basis functions and objects in images is a nontrivial task. Anisotropic basis functions have already been considered and exploited by adaptive e.g. bandelets [6],[7] or non-adaptive e.g. edgelets, wedgelets, curvelets, contourlets, etc. [8]-[16]. These methods build dictionaries of anisotropic basis functions that provide a sparse representation of edges in images.

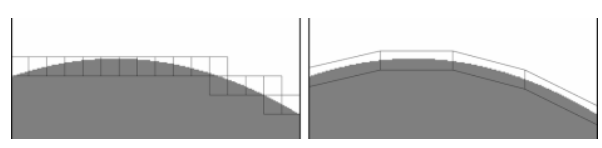

Figure.1. Two types of basis functions representing simple image discontinuity along a smooth curve. (a) Isotropic (b) anisotropic.

Natural edges are not simply stacks of 1-D and they are typically located along smooth curves. Thus, natural images contain intrinsic geometrical structures that are key features in visual information. Because of a separable extension from 1$\mathrm{D}$ bases, wavelets in two-dimensional (2-D) are good at isolating the discontinuities at edge points, but will not capture smoothness along the contours. In addition, separable wavelets can capture only limited directional information. Some notable approaches use non-separable 2-D filter-banks and sub sampling (e.g., quincunx) [17]-[20], but these methods are computationally complex and the design of the associated 2-D filter-banks is often challenging. The advantage with multiscale approach is its inherent implementation, with variable size derivative operators at various scales, to tradeoff between detection and localization of local singularities. The indirect inter-dependence of wavelet coefficients at different scales opens the possibility to explore the hidden association of edge information at various scales (levels) [21]-[24]. In this paper, non-separable and its scaled basis, are applied to provide directional specific 
information along with scale information by allowing directionality with anisotropy and detect the edges in an image.

\section{SKEWED AND ELONGATED BASIS FUNCTIONS FROM DIRECTIONLETS AND EDGE DETECTION}

The standard WT produces isotropic basis functions, which fail to provide a sparse representation of edges and contours. However, directionlets retains the 1-D filtering and subsampling operations and can provide anisotropy.

The Basic Properties of Directionlets:

- Critical sampling

- Simple filter design and Low computational complexity

- Provide anisotropy and multi-directionality

- Basis functions are elongated and oriented in different directions.

- More efficient in edge representation

In addition, the following properties of directionlets are very useful for the edge detection. 1) Multiresolution: The representation should allow images to be successively approximated, from coarse to fine resolutions.

2) Localization: The basis elements in the representation should be localized in both the spatial and the frequency domains.

3) Directionality: The representation should contain basis elements oriented at a variety of directions, much more than the few directions that are offered by separable wavelets.
4) Anisotropy: To capture smooth contours in images, the representation should contain basis elements using a variety of elongated shapes with different aspect ratios.

In the anisotropic wavelet transform, the number of transforms applied along the horizontal and vertical directions is unequal, that is, there are $n_{1}$ horizontal, and $n_{2}$ vertical transforms at a scale, where $n_{1}$ is not necessarily equal to $n_{2}$. Then, the iteration is continued in the low-pass branch, like in the standard wavelet. The anisotropy ratio $\rho=\left(n_{1} / n_{2}\right)$ determines elongation of the anisotropic basis functions. This can be easily implemented by taking inner product between the image data and

a set of basis functions that are essentially skewed and elongated. For example a set of simple basis functions are shown in Fig. 2.

The first level (first scale) basis functions are applied on the image. The basis functions (Fig.2 (a)-(g)) are convolved with an image to get seven sub bands. By combining the all sub bands the edge information in all directions are obtained.

Similarly, the second level basis functions of directionlets provide the seven different sub band images in second scale. The two scale edge information are combined by point by point multiplication commonly known as scale multiplication. The purpose of combining two scales is to suppress noisy components and to enhance the edge information. The combined sub band provides the gradients in the form of edge information that can be used to determine final edge map. In image processing, an edge is often interpreted as one class of singularities characterized easily as discontinuities where the gradient approaches infinity.

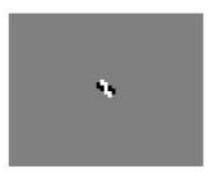

(a)

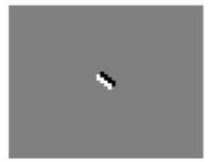

(d)

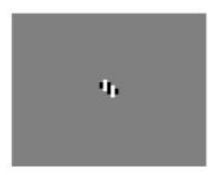

(b)

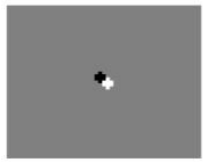

(e)

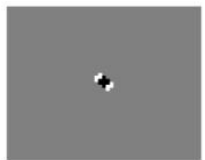

$(\mathrm{g})$

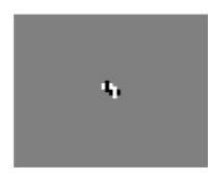

(c)

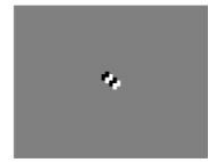

(f)

Figure.2 Seven basis functions for First level Directionlet Transform

However, image data is discrete, edges in an image often are defined as the local maxima of the gradient. In the ideal case, the result of applying an edge labeling algorithm to an image may lead to a set of connected curves that indicate the boundaries of objects, the boundaries of surface markings as well curves that correspond to discontinuities in surface orientation. There are many methods for image edge labeling from its gradients. This paper applied non-maximal suppression (NMS) followed by hysteresis threshold (HT) steps to determine the final edge map. The complete proposed edge detection steps are shown in Fig. 4. 


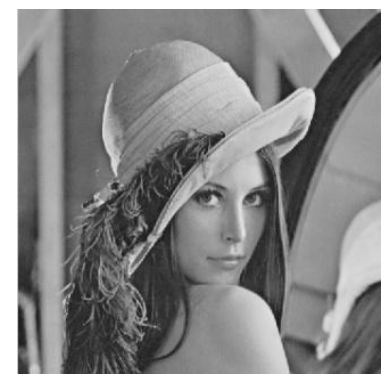

(a)

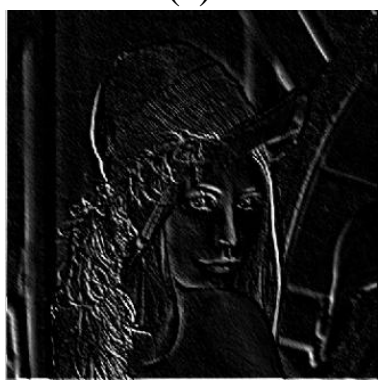

(e)

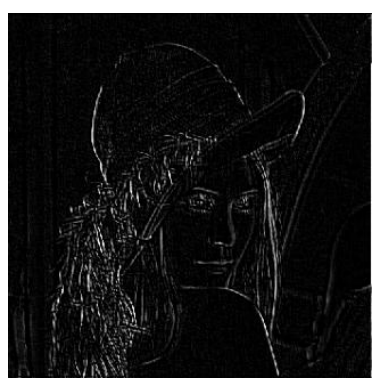

(b)

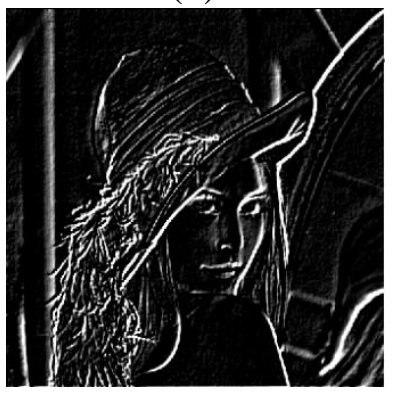

(f)

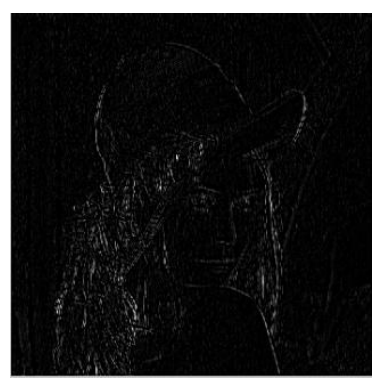

(c)

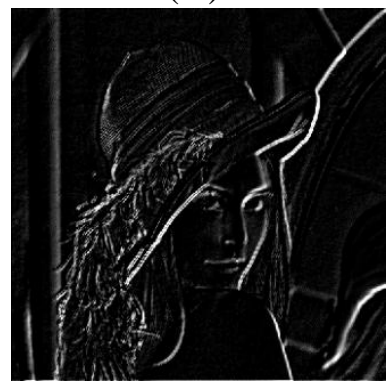

$(\mathrm{g})$

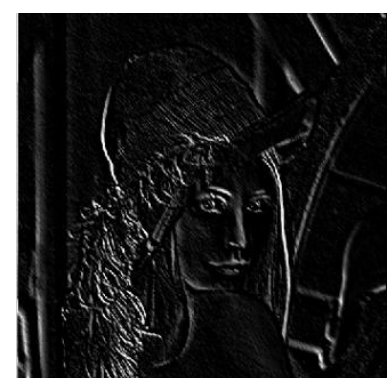

(d)

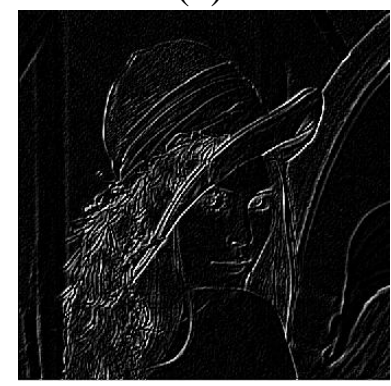

(h)

Figure.3 First level-Seven sub bands of Lena Image (a) Original (b)-(h) Seven sub bands

\subsection{Edge detection algorithm}

To the decomposed image any one of the following edge detection techniques are used to obtain the edge detected result. Edge detection techniques used are as follows.

\subsubsection{Gray thresholding}

The gray threshoding is the simplest and most widely used thresholding method. It computes a global threshold that can be used to convert an intensity image to a binary image. This technique uses Otsu's method, which chooses the threshold to minimize the intra class variance of the black and white pixels

\subsubsection{Adaptive thresholding}

Due to uneven illumination and other factors, the background gray level and contrast between the objects and background often vary within the image. In such cases, global threshold is unlikely to produce satisfactory results, since a threshold that works well in one area of the image might be poor in other areas. To cope up this variation, adaptive or variable thresholding is used.

Implementing adaptive threshold is by two pass operation. Before the first pass, a threshold is computed based on the histogram of each block by choosing. In the first pass the object boundaries are defined using a gray level threshold that is constant within each block but differs for various blocks. On the second pass, each object is given its own threshold that lies midway between its interior gray level and background.

\subsubsection{Non maximal suppression}

Non-maximum suppression is often used along with edge detection algorithms. The image is scanned along the image gradient direction, and if pixels are not part of the local maxima they are set to zero. This has the effect of suppressing all image information that is not part of local maxima. Given estimates of the image gradients, a search is then carried out to determine if the gradient magnitude assumes a local maximum in the gradient direction.

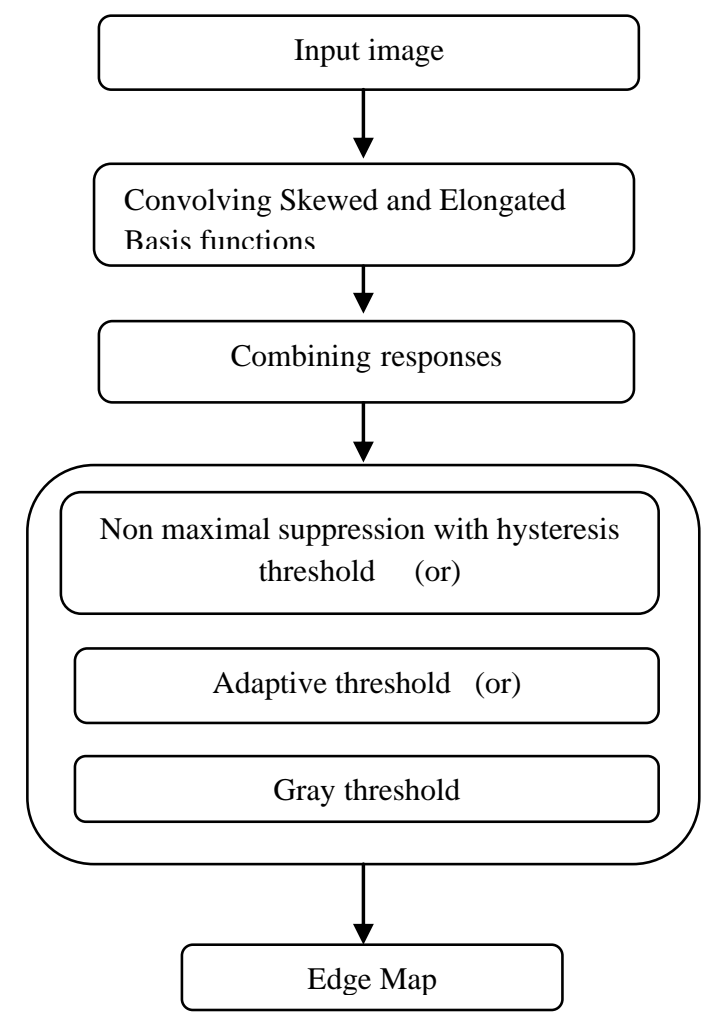

Figure.4 Block diagram of the proposed method

\section{RESULTS AND DISCUSSIONS}

The proposed method has been tested on the 'Lena' image of size 512 × 512 Fig. 5 (a), 'Building' image of size 463 × 651 Fig.6 (a) and 'Series' image of size 540 x 569 Fig.7 (a). Fig.5(c)-(f) shows the edge labeled of scale multiplied image Fig.5 (b), using NMS followed by HT and is compared with classical Canny, Sobel and Prewitt and shown in Fig 5(d)-(f) respectively. NMS is often used along with edge detection algorithms. 


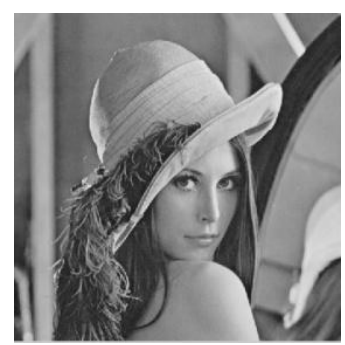

(a)

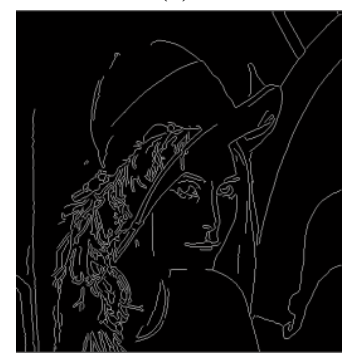

(d)

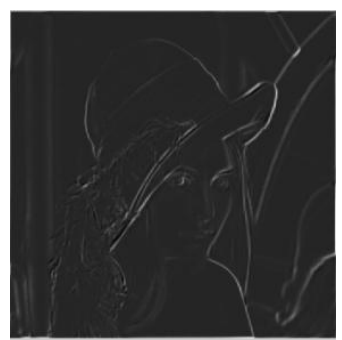

(b)

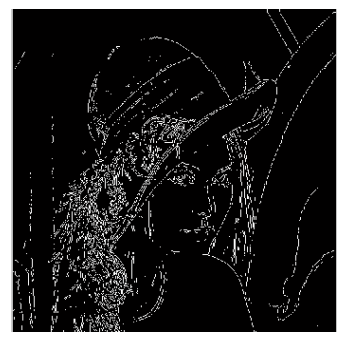

(e)

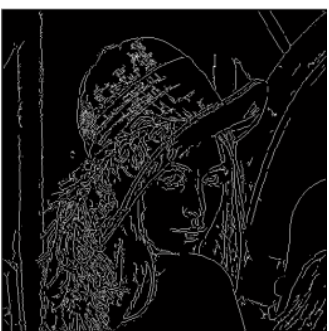

(c)

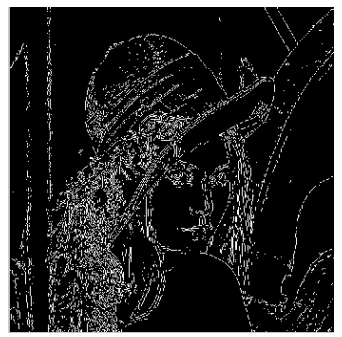

(f)

Figure 5. Edge Detection Comparison with conventional operator (a) Original (b) Scale multiplied (c) Proposed(NMS+HT) (d) Canny (e) Sobel (f) Prewitt

The image is scanned along the image gradient direction, and if pixels are not part of the local maxima, they are set to zero. This has the effect of suppressing all image information that is not part of local maxima. Given estimates of the image gradients, a search is then carried out to determine if the gradient magnitude assumes a local maximum in the gradient direction. From the Fig. 5, it is observed that the proposed method detected more edge features than the conventional Canny, Sobel and Prewitt operator. The algorithms tested on various images are shown in Fig.6 and Fig.7.

We compare the performances of the edge detection by our methodology using various parameters true positive (TP), false positive (FP), true negative (TN), false negative (FN) [26], [27] and Figure of Merit ' $F$ ' [28] with that of the conventional edge detectors like Canny, Sobel, Prewitt. In our experiments, the canny edge detector uses two thresholds; lower threshold and upper threshold as 0.1 and 0.25 respectively. The standard deviation is set to one. The optimal setting is made according to the canny method in [25] that is being used in our experiment. For every pixel in an image there are four possible edge detection results. The edge pixel is compared with the ground truth image to detemine the accuracy, sensitivity, and selectivity. Fig 8(a), 8(b) and Fig 8(c) shows the original, corresponding ground truth and proposed edeg detection output. The true positive rate (TPR) or sensitivity is given by $\mathrm{TPR}=\frac{\mathrm{TP}}{\mathrm{TP}+\mathrm{FN}}$. False positive rate $(F P R)$ is defined as $\mathrm{FPR}=\frac{\mathrm{FP}}{\mathrm{FP}+\mathrm{TN}}$. True negative rate (TNR) or Specificity is given by TNR $=1-\mathrm{FP}$. Accuracy in percentage is obtained by $\mathrm{ACC}=\frac{\mathrm{TP}+\mathrm{TN}}{\mathrm{TP}+\mathrm{TN}+\mathrm{FP}+\mathrm{FN}} \times 100$. Where TP-Edge pixel in an image is detected correctly as an edge pixel, FP-Non edge pixel is detected wrongly as edge pixel, TN-Non edge pixel detected correctly as non edge pixel, and FN-Edge pixel detected wrongly as non edge pixel. The figure of merit is given by $F=\frac{1}{[\max \{N i, N a\}]} \sum_{k=1}^{N d} \frac{1}{1+\alpha d^{2}(k)} \quad$.The performances are evaluated using the above formulas and is tabulated in the Table 1. Figure of merit comparison is shown in the Table 2.

It is observed that the accuracy, figure of merit of the proposed methodology is higher compared to the conventional edge detectors and also the sensitivity is low and the specificity is high compared to other classical edge detectors hence providing better performance in terms of accuracy 


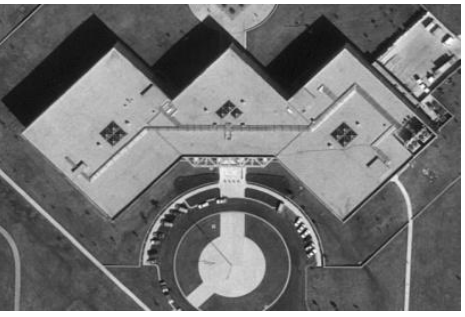

(a)

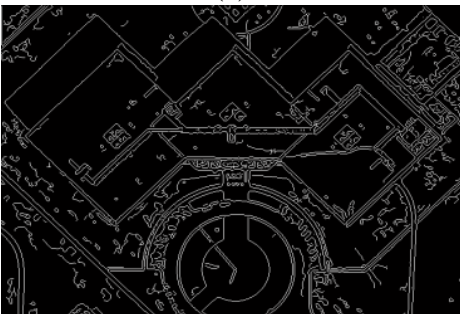

(d)

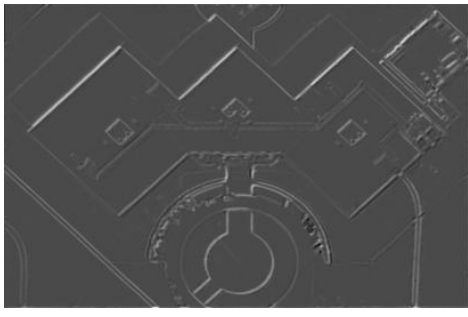

(b)

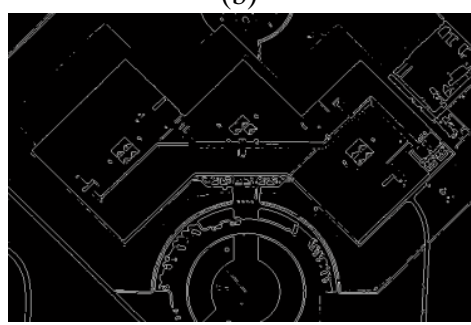

(e)

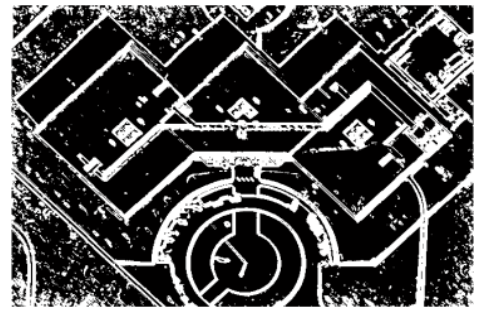

(c)

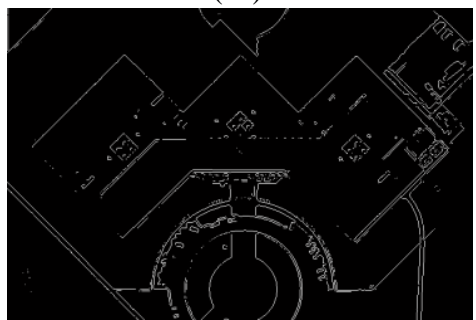

(f)

Figure.6. Edge Detection Comparison with conventional operator (a) Original 'Building'

(b) Scale multiplied image (c) Proposed(Adaptive) (d) Canny (e) Sobel (f) Prewitt

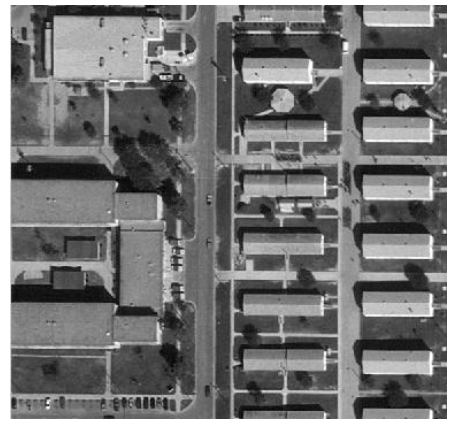

(a)

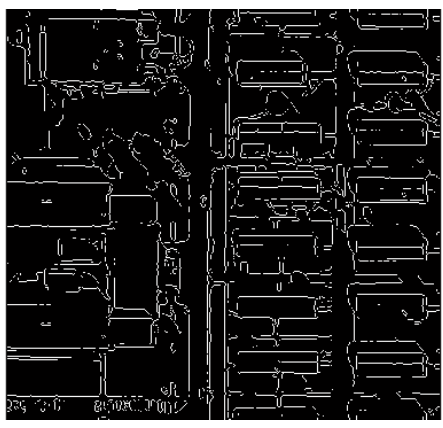

(d)

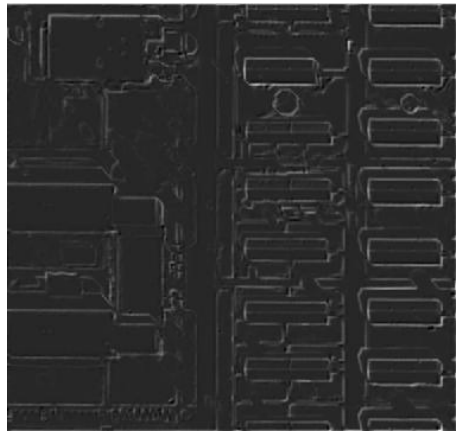

(b)

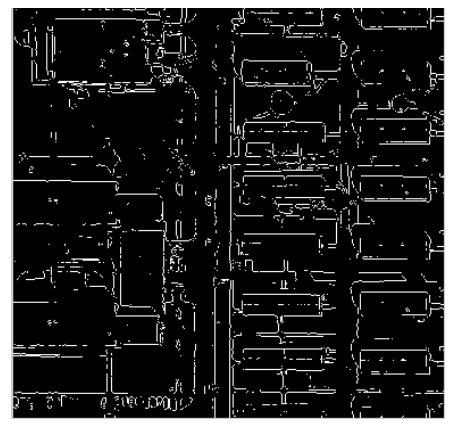

(e)

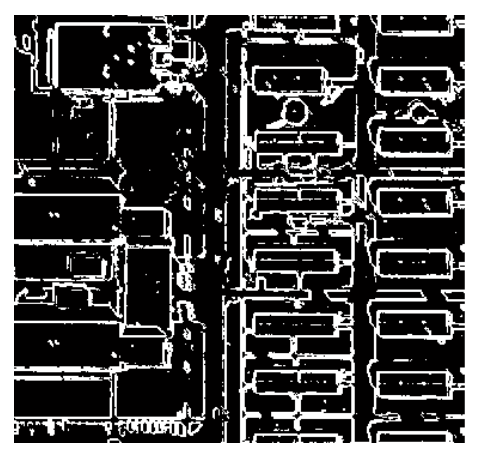

(c)

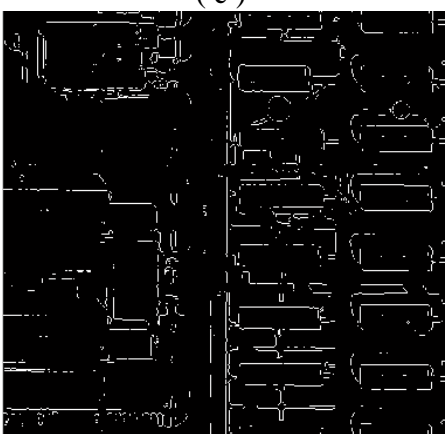

(f)

Figure.7. Edge Detection Comparison with conventional operator (a) Original 'Serial' image

(b) Scale multiplied image (c) Proposed(Gray) (d) Canny (e) Sobel (f) Prewitt

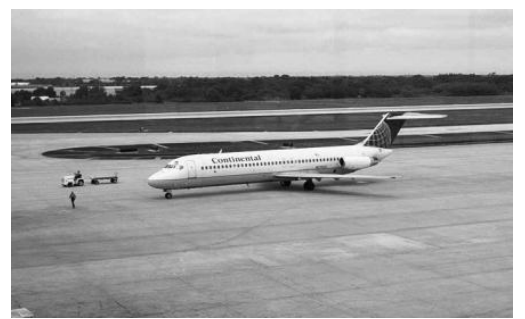

(a)

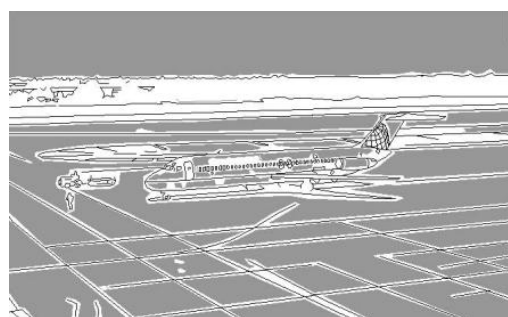

(b)

Figure. 8. (a) original airplane image (b) ground truth 
Table1. Comparison between proposed methodology and various edge detection algorithms

\begin{tabular}{ccccccc}
\hline & $\begin{array}{c}\text { SEBF+ } \\
\text { NMS+HT }\end{array}$ & SEBF+GT & SEBF+AT & Sobel & Canny & Prewitt \\
\hline Parameters & 3495 & 5050 & 6349 & 10841 & 6066 & 6722 \\
\hline FN & 10145 & 8590 & 7291 & 2799 & 7574 & 6918 \\
\hline FP & 7723 & 13702 & 20929 & 68954 & 22167 & 70684 \\
\hline TN & 248168 & 242189 & 234962 & 186937 & 233724 & 185207 \\
\hline TPR (Sensitivity) & 0.2562 & 0.3702 & 0.4655 & 0.7948 & 0.4447 & 0.4928 \\
\hline FPR & 0.0302 & 0.0535 & 0.0818 & 0.2695 & 0.0866 & 0.2762 \\
\hline TNR (Specificity) & 0.9698 & 0.9465 & 0.9182 & 0.7305 & 0.9134 & 0.7238 \\
\hline Accuracy (in & 93.37 & 91.73 & 89.53 & 73.38 & 88.97 & 71.21 \\
\hline
\end{tabular}

Table 2.Figure of Merit ' $F$ ' comparison between proposed methodology and various algorithms

\begin{tabular}{cc}
\hline Methods & Figure of merit ' $F$ ' \\
\hline $\begin{array}{c}\text { SEBF+ NMS+HT } \\
\text { (Proposed) }\end{array}$ & 0.7480 \\
\hline SEBF+GT & 0.6695 \\
\hline SEBF+AT & 0.6599 \\
\hline Canny & 0.6051 \\
\hline Sobel operator & 0.5794 \\
\hline Prewitt operator & 0.3602 \\
\hline
\end{tabular}

\section{CONCLUSION}

In this paper, we have proposed the Skewed and Elongated Basis functions (SEBF) based edge detection which is useful to capturing and representing singularities in various orientations and scales. This approach helps in providing directional specific information along with scales hence collecting more information. This study compares the work with that of Sobel edge operator, Canny operator and Prewitt edge operator. In addition, our proposed methodology has provided better results in terms of accuracy, sensitivity, specificity, and figure of merit $(\mathrm{F})$. From the qualitative and quantitative results it is observed that the proposed method for edge detection provides a better result and acts as the accurate method of extracting the information along the edges.

\section{REFERENCES}

[1] Mallat S, HW ANG W L 2002 .Singularity Detection and Processing with Wavelets. IEEE Trans on Info Theory, 617-643

[2] Mitra Basu, 2002. Gaussian-Based Edge-Detection Methods-A Survey. IEEE Transactions on Systems, Man, and Cybernetic, 252-260

[3] Castleman K. R., 1996. Digital Image Processing. Englewood Cliffs, NJ: Prentice-Hall

[4] Donoho D. L., Vetterli M., DeVore R. A., and Daubechies I., 1998. Data compression and harmonic analysis. IEEE Trans. Inf. Theory, 2435-2476 
[5] Vetterli M. and Kova`cevic' J.,1995. Wavelets and Subband Coding. EnglewoodCliffs, NJ: Prentice-Hall

[6] Pennec E. L. and Mallat S.,2000. Image compression with geometric wavelets. IEEE Int. Conf. Image Processing, 661-664

[7] Stephane Mallat,Gabriel Peyre 2005. Sparse geometric image representations with bandelets, IEEE Trans. Image Process.,. 423-438.

[8] Donoho D. L.,1999. Wedgelets: Nearly-minimax estimation of edges. Ann.Statist., 859-897

[9] Romberg J. K.,.Wakin M, and Baraniuk R.,2002. Multiscale wedgelet image analysis: Fast decompositions and modeling. IEEE Int. Conf. Image Processing, 585588

[10] Romberg J. K., Wakin M., and Baraniuk R 2003. Approximation and compression of piecewise smooth images using a wavelet/wedgelet geometric model. IEEE Int. Conf. Image Processing, Barcelona, Spain, 49-52.

[11] Wakin M., Romberg J., Hyeokho C., and Baraniuk R.,2002. Rate-distortion optimized image compression using wedgelets. IEEE Int. Conf. Image Processing, 237 -240 .

[12] Wakin M., Romberg J., Choi H., and Baraniuk R., 2006. Wavelet-domain approximation and compression of piecewise smooth images. IEEE Trans. Image Process, 1071-1087

[13] Candès E. J. and Donoho D. L.,1999. Curvelets - A surprisingly effective nonadaptive representation for objects with edges, in Curve and Surface Fitting, A. Cohen, C. Rabut, and L. L. Schumaker, Eds. Nashville, TN: Vanderbilt Univ. Press

[14] Candès E. J. and Donoho D., 1999. Curvelets and curvilinear integrals, Tech. Rep., Dept. Statist., Stanford Univ., Stanford, CA

[15] Candès E. J. and Donoho D. L. 2002. New tight frames of curvelets and optimal representations of objects with smooth singularities, Tech. Rep., Dep. Statist., Stanford Univ., Stanford, CA
[16] Do M. N. and Vetterli M.,2005. The contourlet transform: An efficient directional multiresolution image representation, IEEE Trans. Image Process., 2091-2106

[17] Skodras A., Christopoulos C., and Ebrahimi T., 2001. The JPEG 2000 still image compression standard, IEEE Signal Processing Mag., 36-58

[18] Cohen A. and Daubechies I.,1993. Non-separable bidimensional wavelet bases, Revista Mat. Iberoamer., 51-137

[19] J.Kova“cevic' 1991, Filter Banks andWavelets: Extensions and Applications," Ph.D. dissertation, Graduate School of Arts and Sciences, Columbia Univ., New York.

[20] Kova`cevic' J. and Vetterli M.,1992. Nonseparable multidimensional perfect reconstruction filter banks and wavelet bases for , IEEE Trans. Inf. Theory, 533-555

[21] Rosenfeld A, and Thurston M, 1970. Edge and Curve Detection for Visual Scene Analysis, IEEE Trans. Computer,562-569

[22] Sadler B M, Pham T, and Sadler L C, 1998. Optimal and wavelet based Shock Wave Detection and Estimation, J Acoust. Soc. Am., 955-963

[23] Ziou D, and Tabbone S, 1993.A Multiscale Edge Detector, PR, 26(9), 1305-1314

[24] Park D J, Nam K N, and Park R H, 1995.Multiresolution Edge Detection Techniques, Pattern Recognition, 211219

[25] Canny J., 1986.A computational approach to edge detection, IEEE Trans. Pattern Anal. Mach. Intell., 679698

[26] Bowyer K., Kranenburg C., and Dougherty S.,2001. Edge detector evaluation using empirical ROC curves,Comput. Vis. Image Underst 77-103

[27] Wei Jiang, Kin-Man Lam, and Ting-Zhi Shen,2009. Efficient Edge Detection Using Simplified Gabor Wavelets, IEEE Transactions On Systems, Man, and Cybernetics- Part B: Cybernetics

[28] Pratt, W.K.2007. Digital Image Processing, (John Wiley \& Sons, New York, Fourth Edition. 